\title{
Primary Omental Torsion: Two Case Reports
}

\author{
Ibtisam Al-Bader ${ }^{a}$ Ali Al-Said Ali ${ }^{a}$ Khalid Al-Sharraf ${ }^{a}$ Abdulla Behbehani ${ }^{b}$ \\ ${ }^{a}$ Department of General Surgery, Mubarak Al-Kabeer Hospital, and ${ }^{b}$ Faculty of Medicine, Kuwait University, Kuwait
}

\section{Key Words}

Abdominal pain $\cdot$ Omental torsion $\cdot$ Omental infarction

\begin{abstract}
Objective: To describe two cases of primary omental torsion as an uncommon cause of abdominal pain. Clinical Presentation and Intervention: Two female patients presented to our surgical service with right-sided abdominal pain. In view of their clinical presentation and workup, both patients required operative intervention. At laparotomy, the cause of the pain was recognized to be due to infarcted omentum secondary to torsion. Both patients underwent omentectomy and appendectomy and had an uneventful recovery. Conclusion: Primary torsion of the omentum is difficult to diagnose preoperatively; this condition is usually detected during surgical exploration for acute abdominal pain.
\end{abstract}

Copyright @ 2007 S. Karger AG, Basel

\section{Introduction}

Primary torsion of the greater omentum is an uncommon cause of abdominal pain. The diagnosis is usually made intraoperatively [1]. Resection of the infarcted omentum is the treatment of choice. We report two cases of omental torsion to describe this more frequently encountered condition, and its management.

\section{Case Reports}

Case 1

A 55-year-old female presented with a history of lower abdominal pain of 2 days' duration. The pain started in the epigastric area; $2 \mathrm{~h}$ later, it shifted to the right iliac fossa. It was colicky in nature, without any radiation. There was no associated nausea or vomiting. On examination, a lower midline incision due to a previous cesarean section was noted. She was in pain, with stable vital signs, and a temperature of $37^{\circ} \mathrm{C}$. Examination of the abdomen demonstrated mild cough tenderness in the right iliac fossa, and mild rebound tenderness.

Laboratory investigation revealed a white cell count of $8.6 \times$ $10^{9} / 1$, coagulation studies, liver and renal function tests were within normal limits except for mildly elevated alkaline phosphatase. Ultrasound scan of the abdomen did not reveal any intraabdominal pathology. A presumptive diagnosis of acute appendicitis was made.

After induction of anesthesia and at surgery, a mass measuring $4 \times 4 \mathrm{~cm}$ was palpable in the right lumbar region. Through a lower midline incision, $300 \mathrm{ml}$ of clear serous fluid, and a gangrenous piece of omentum measuring $3 \times 4 \mathrm{~cm}$ were noted (fig. 1). The appendix was normal. No intra-abdominal adhesion was present. Omentectomy and appendectomy were carried out. Postoperative recovery was uneventful, and the patient was discharged on the sixth postoperative day.

Case 2

A 48-year-old diabetic female presented with a history of abdominal pain of 2 days' duration. The pain was localized to the right upper quadrant without radiation. It was colicky in nature and of moderate severity. There was no history of nausea or vomiting. She had had an abdominoplasty 5 years ago. Examination

\section{KARGER}

Fax +41613061234

E-Mail karger@karger.ch

www.karger.com
(C) 2007 S. Karger AG, Basel

$1011-7571 / 07 / 0162-0158 \$ 23.50 / 0$

Accessible online at:

www.karger.com/mpp
Dr. Ibtisam Al-Bader

Department of Surgery

Mubarak Al-Kabeer Hospital

Safat 13110 (Kuwait)

Tel. +965986 4180, E-Mail albader_ib@hotmail.com 


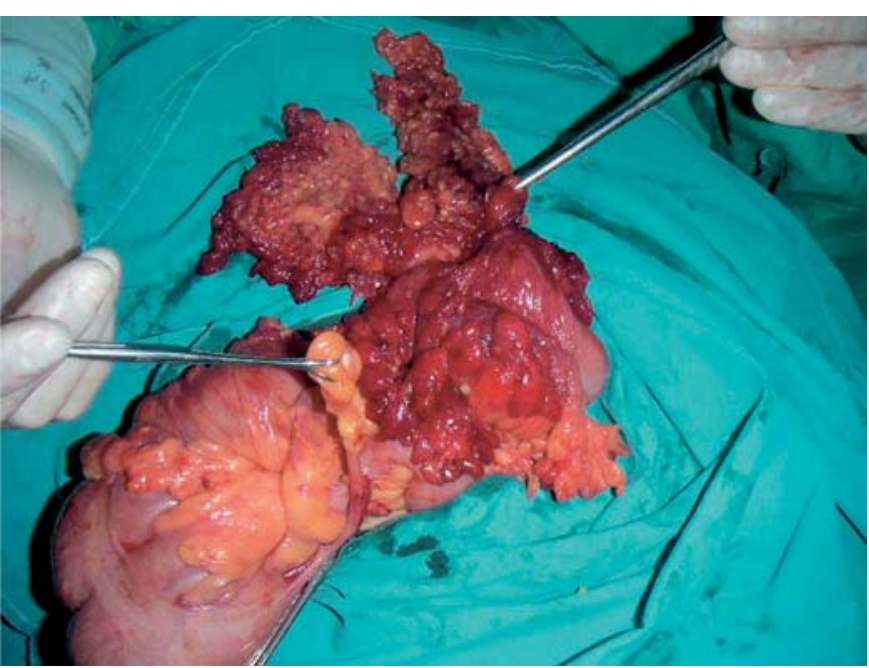

Fig. 1. Infarcted portion of the omentum is congested and hemorrhagic.

revealed that the patient was in pain, with a temperature of $37.2^{\circ} \mathrm{C}$. Abdominal examination revealed the presence of right upper quadrant tenderness. Chest X-ray failed to reveal any air under the diaphragm. Laboratory investigations revealed a white cell count of $12.4 \times 10^{9} / 1$, liver and renal function tests were within normal limits and serum amylase was $105 \mathrm{U} / 1$. Abdominal ultrasound scan was normal.

The patient continued to have abdominal pain, the intensity of which gradually increased. CT scan of the abdomen, with oral and intravenous contrast (fig. 2), revealed retrocecal inflammatory fat strands with localized inflammatory changes seen around the cecum. At surgery, through a Lanz incision, clear serous peritoneal fluid, a gangrenous, twisted omentum adherent to the hepatic flexure of the colon and a normal appendix were noted. No intra-abdominal adhesion was present. Omentectomy and appendectomy were carried out through the same incision. The patient's postoperative recovery was uneventful from the surgical point of view. The patient was discharged on the fifth postoperative day.

\section{Discussion}

Primary idiopathic torsion of the omentum is an unusual cause of acute abdominal pain. The diagnosis is rarely made preoperatively [1]. Primary omental torsion is diagnosed when laparotomy fails to reveal any other significant intra-abdominal pathology to account for the presenting complaint. This occurs when the omentum twists along its longitudinal axis with subsequent compromise of the vascular supply [1]. Obesity has been postulated as a cause of primary omental torsion. However,

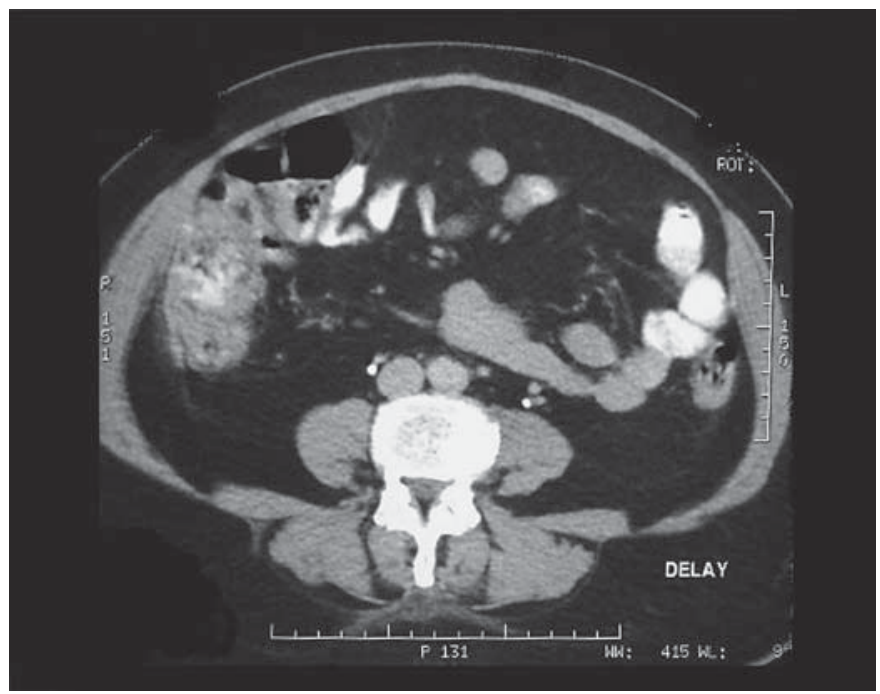

Fig. 2. Retrocecal inflammatory fat stranding.

it was found that only $12 \%$ of patients with primary omental torsion were obese [2].

Secondary omental torsion is more common than the primary type, and occurs in association with adhesions, cysts, tumors, and external or internal hernias [3].

The main symptoms and signs are usually nonspecific and localized in the right side of the abdomen, particularly the right lower quadrant. Left-sided omental torsion occurs less frequently [4].

Associated symptoms include nausea, vomiting and anorexia in $25 \%$ of the patients. The body temperature may be normal or slightly elevated. Tenderness in the right iliac fossa is a common finding on examination and a mass is noted in one third of the patients [5]. Laboratory investigations may show moderate leukocytosis. In most cases, a presumptive diagnosis of acute appendicitis or acute cholecystitis is made as in case 1.

Diagnostic imaging with ultrasonography and CT scanning may be useful but are not very reliable. These modalities may aid in establishing the diagnosis preoperatively with evidence of diffuse streaking of the greater omentum and a mass of fat density with a characteristic concentric distribution of fibrous and fatty folds converging radially towards the site of the torsion $[4,6]$.

Ultimately, surgical exploration is the most appropriate diagnostic and therapeutic modality in such patients as in our 2 cases. Free fluid, usually serosanguineous, is found in the abdominal cavity. However, both our pa- 
tients had clear intraperitoneal fluid. Resection of the involved segment of the omentum is recommended $[1,3]$.

Although spontaneous recovery of omental infarction has been reported, expectant management carries the risk of abscess formation which may result in prolongation of the abdominal pain and hospital stay $[1,7]$. Laparoscopy for diagnosis and resection of the necrotic omentum has been reported $[7,8]$. Laparoscopy can be utilized as a diagnostic as well as therapeutic modality in the management of this uncommon condition $[7,8]$.

\section{Conclusion}

Primary torsion of the omentum is difficult to diagnose preoperatively; this condition is usually detected during surgical exploration for acute abdominal pain.

\section{References}

-1 Karayiannakis AJ, Polychronidis A, Chatzigianni E, Simopoulos C: Primary torsion of the greater omentum: report of a case. Surg Today 2002;32:913-915.

$\checkmark 2$ Mainzer R, Simoes A: Primary idiopathic torsion of the omentum - Review of the literature and report of six cases. Arch Surg 1964;88:974-983.

3 Haldar PJ: Primary omental torsion: case report. Trop Gastroenterol 2000;21:207.
4 Aoun N, Haddad-Zebouni S: Left-sided omental torsion: CT appearance. Eur Radiol 2001;11:96-98.

5 Natarajan A, Rozario A: Omental torsion with acute appendicitis: an interesting association. Trop Doct 2002;32:243-244.

-6 Schwartzman GJ, Jacobs JE, Birnbaum BA: Omental infarction as a delayed complication of abdominal surgery. Clin Imaging 2001;25:341-343.
7 Sanchez J, Rosado R, Ramirez D: Torsion of the greater omentum: treatment by laparoscopy. Surg Laparosc Endosc Percutan Tech 2002;12:443-445.

8 Goti F, Hollman R, Stieger R: Idiopathic segmental infarction of the greater omentum successfully treated by laparoscopy: report of a case. Surg Today 2000;30:451-453. 